\title{
Steady-State Queue Length Analysis of a Batch Arrival Queue under N-Policy with Single Vacation and Setup Times
}

\author{
Zhong Yu ${ }^{1}$, Mingwu Liu ${ }^{2}$, Yongkai Ma ${ }^{1}$ \\ ${ }^{1}$ School of Management and Economics, University of Electronic Science and Technology of China, Chengdu, China \\ ${ }^{2}$ School of Management, Chongqing Jiaotong University, Chongqing, China \\ E-mail: liumingwu2007@yahoo.cn
}

Received March 10, 2010; revised April 20, 2010; accepted May 23, 2010

\begin{abstract}
This paper investigates the steady state property of queue length for a batch arrival queue under N-policy with single vacation and setup times. When the system becomes empty, the server is turned off at once and takes a single vacation of random length $V$. When he returns, if the queue length reaches or exceeds threshold $N(N \geq 1)$, the server is immediately turned on but is temporarily unavailable due to a random setup time $U$ before offering service. If not, the server stays in the system until the queue length at least being $N$. We derive the system size distribution and confirm the stochastic decomposition property. We also derive the recursion expressions of queue length distribution and other performance measures. Finally, we present some numerical examples to show the analytical results obtained. Sensitivity analysis is also performed.
\end{abstract}

Keywords: Queue Length, Recursion Expressions, N Policy, Setup

\section{Introduction}

This paper pays attention to a batch arrival queueing system under N-policy with a single vacation and setup times, which can model queue-like manufacturing/production/inventory system. Consider a system of processing in which the operation does not start until some predetermined number $(N)$ of semi-finished products waiting for processing. To be more realistic, the machine need a setup time for some preparatory work before starting processing. When all the semi-finished products in the system are processed, the machine is shut down and leaves for a vacation. The operator performs machine repair, preventive maintenance and some other jobs during the vacation. After these extra operations, the operator returns and checks the number of semi-finished products in the queue determining whether or not start the machine.

Queueing system with vacations has been attracted considerable attention to many authors. It has effectively been applied in computers and communication systems,

The authors also thank the National Nature Science Foundation of China for its support (contract: 70672104). production/inventory system. Doshi [1] and Takagi [2] presented an excellent survey of queueing system with server vacations. One of the important achievements for vacation queueing system is the famous stochastic decomposition results, which was first established by Fuhrmann and Cooper [3].

The N-policy was first introduced by Yadin and Naor [4], which is a control policy turning the server on whenever $\mathrm{N}$ (a predetermined value) or more customers in the system, turning off the server when system is empty. Lee et al. [5] successfully combined the batch arrival queue with $\mathrm{N}$-policy and obtained the analytical solutions. Later, Lee et al. [6,7] analyzed in detail a batch arrival $\mathrm{M}^{\mathrm{x}} / \mathrm{G} / 1$ queue under $\mathrm{N}$-policy with a single vacation and repeated vacation respectively. They derived the system size distribution which confirmed the famous stochastic decomposition property, and the optimal stationary operating policy was also investigated. At the present day, batch arrival queueing system under $\mathrm{N}$-policy with different vacation policies have been received considerable attention because of its practical implication in production/inventory system. A number of searchers, such as Choudhury et al. [8-10], Ke [11,12], and Reddy et al. [13], and many authors not be listed 
above have considered batch arrival queueing system under $\mathrm{N}$-policy with various vacation policies.

It is to be noted that few authors involved above considered the probability distribution of the number of customers in the system for batch arrival queue under $\mathrm{N}$-policy with different vacations policies. Recently, Wang et al. [14] analyzed the behaviors of queue length distributions of a batch arrival queue with server vacations and breakdowns based on a maximum entropy approach $[15,16]$. Ke et al. [17] also used the maximum entropy solutions for batch arrival queue with an un-reliable server and delaying vacations. They all derived the approximate formula for the probability distribution of the number of customers in the system. Tang and his co-author $[18,19]$ paid attention to the queue length distribution for queueing system, because it is an important performance measure for the system design and optimization. For example, the queue-length distribution has been applied for the communication system buffer design [20].

To the best of our knowledge, fewer researchers have derived the queue length distribution under $\mathrm{N}$-policy batch arrival queueing system. Although, the batch arrival under N-policy have been studied extensively, but the exact analytical solutions for queue length distribution do not be obtained. This motives us to develop an approach for the queue length distribution of the N-policy batch arrival queueing system. In this paper, we study the steady state queue length for an $\mathrm{M}^{\mathrm{x}} / \mathrm{G} / 1$ under $\mathrm{N}$ policy with single vacation and setup times. First, we derive the system size distribution by the supplementary variables method. Second, using the Leibniz formula of derivation combing some knowing results, we obtain the additional queue length distribution and the recursion expressions of the queue length distribution. Finally, we present several examples for application of these recursion expressions. Also, the effect of different system parameters on the queue length distribution is investigated.

\section{The Mathematical Model and Notations}

This paper considers an $\mathrm{M}^{\mathrm{x}} / \mathrm{G} / 1$ queueing system where the arrival occurs according to a compound Poisson process with random batch size $X$. Arriving customers in the queue form a single waiting line and the service discipline is assumed to be FCFS. The service time is an independent and identically distributed random variable with a general distribution function $S(t), t \geq 0$, and with finite mean service time. The server can only process one customer at a time. The server is turned off each time if there is no customer in the queue and leaves for a vacation of random length $V$. When he returns from the vacation and finds the queue length is no less than $N$, the server starts to setup with random length $U$. Other- wise, he stays in the system and does not start setup until the customers reaches and exceeds $N$. When the setup is completed, the server begins to serve the customers until there is no customer in the system.

Throughout the analysis, the following notations and the variables will be adopted.

$$
\begin{aligned}
& N=\text { threshold }(N \geq 1) \\
& \lambda=\text { mean arrival rate } \\
& \mu=\text { mean service rate } \\
& E[V]=\text { mean vacation time } \\
& E[U]=\text { mean setup time } \\
& S=\text { service time random variable } \\
& V=\text { vacation times random variable } \\
& U=\text { setup times random variable } \\
& S(x)=\text { the probability density function } S \\
& v(x)=\text { the probability density function } V \\
& u(x)=\text { the probability density function } U \\
& S^{*}(\theta)=\text { the laplace-Stieltjies transform (LST) of } S \\
& V^{*}(\theta)=\text { the LST of } V \\
& \hat{U}^{*}(\theta)=\text { the LST of } U \\
& S^{0}(t)=\text { remaining service time of the customer in ser- }
\end{aligned}
$$
vice at time $t$

$V^{0}(t)=$ remaining vacation time of the customer in vacation at time $t$

$U^{0}(t)=$ remaining setup time of the customer in setup at time $t$

$N(t)=j$, the number of customers in the queue at time $t$

$\alpha_{k}=$ probability that $k$ customers arrival during a vacation

$$
\begin{aligned}
& g_{k}=P(X=k), \text { where } k=1,2, \cdots \\
& X(z)=\sum_{k=1}^{\infty} g_{k} z^{k} \text {, the p.g.f. of each batch size } \\
& Y(t)=0 \text { if the server is on vacation } \\
&=1 \text { if the server is in dormancy } \\
&=2 \text { if the server is in setup } \\
&=3 \text { if the server is busy }
\end{aligned}
$$

$\rho=$ traffic intensity, $\rho=\lambda E[X] / \mu$. In the steady state $\rho$ should be assumed to be less than unity $m[k]=m_{1}+\cdots+m_{k}$, the number of customers presents the sum of $k$ batches of customers

$$
\sum_{m[k]=j-1}=\sum_{m_{1}=1}^{i_{1}} \cdots \sum_{m_{k}=1}^{i_{k}} \text { and } i_{1}+\cdots+i_{k}=j-1 \text {, the sum of }
$$

$k$ batches of customer equals to $j-1$. 


\section{Preliminary Formula for $\mathbf{M}^{\mathrm{x}} / \mathrm{G} / \mathbf{1}$ Queueing System}

Before discussing our model, let us recall some results in the ordinary $\mathrm{M}^{\mathrm{x}} / \mathrm{G} / 1$ queueing system. Let $\tilde{p}_{j}^{0}=\lim _{t \rightarrow \infty} P$. $\{N(t)=j\}, j \geq 0$ be the steady state queue length distribution for the $\mathrm{M}^{\mathrm{x}} / \mathrm{G} / 1$ queueing system. From Tang et al. [19], the recursion expressions of queue-length distribution are as follows:

$$
\begin{gathered}
\tilde{p}_{0}^{0}=1-\rho \\
\tilde{p}_{j}^{0}=\lambda(1-\rho)\left\{\theta_{j}+\sum_{s=1}^{j-1} \theta_{j-s}\left[1-\sum_{i=1}^{s} g_{i}\right]\right\}, j \geq 1
\end{gathered}
$$

where

$$
\begin{gathered}
\theta_{1}=\frac{1-s(\lambda)}{\lambda s(\lambda)} ; s(\lambda)=\int_{0}^{\infty} e^{-\lambda t} d S(t) ; \\
\theta_{j}=\frac{1}{s(\lambda)}\left\{\sum _ { i = 1 } ^ { j - 1 } \sum _ { m [ i ] = j - 1 } g _ { m _ { 1 } } \cdots g _ { m _ { i } } \int _ { 0 } ^ { \infty } e ^ { - \lambda t } \left[1-S(t) \frac{(\lambda t)^{i}}{i !} d t\right.\right. \\
\left.+\sum_{i=1}^{j-1} \theta_{j-1}\left[1-s(\lambda)-\sum_{i=1}^{i} \sum_{m[k]=k}^{i} g_{m_{1}} \cdots g_{m_{k}} \int_{0}^{\infty} e^{-\lambda t} \frac{(\lambda t)^{k}}{k !} d S(t)\right]\right\}
\end{gathered}
$$$$
j \geq 1 \text {. }
$$

Let $G(z)$ be the p.g.f. of the number of customers in the queue at stationary. We have

$$
G(z)=\frac{(1-\rho)(1-z) S^{*}(\lambda-\lambda X(z))}{S^{*}(\lambda-\lambda X(z))-z}
$$

\section{The System Size Distribution}

This section, we set up the system equation for the system size distribution at stationary and derive the p.g.f. of the system size distribution. We introduce the supplementary variables $S^{0}(t), V^{0}(t)$ and $U^{0}(t)$ for obtaining a Markov process $\{N(t), \delta(t)\}$, where $\delta(t)=V^{0}(t)$ if $Y(t)=0, \delta(t)=0$, if $Y(t)=1, \delta(t)=U^{0}(t)$, if $Y(t)=2$ and $\delta(t)=S^{0}(t)$, if $Y(t)=3$. Denote

$$
\begin{gathered}
Q_{n}(x, t) d t \equiv P\left[N(t)=n, x \leq V^{0}(t) \leq x+d t, Y(t)=0\right] \\
n=0,1, \cdots
\end{gathered}
$$

$$
\begin{gathered}
R_{n}(t) \equiv P[N(t)=n, Y(t)=1], n=0, \cdots, N-1 \\
U_{n}(x, t) d t \equiv P\left[N(t)=n, x \leq U^{0}(t) \leq x+d t, Y(t)=2\right] \\
n=0,1, \cdots \\
P_{n}(x, t) \equiv P\left[N(t)=n, x \leq S^{0}(t) \leq x+d t, Y(t)=3\right] \\
n=0,1, \cdots
\end{gathered}
$$

Following the argument of Lee et al. [5-7], we can easily set up the following steady state system equations the supplementary variables technique.

$$
\begin{gathered}
-\frac{d}{d x} P_{1}(x)=-\lambda P_{1}(x)+P_{2}(0) s(x) \\
-\frac{d}{d x} P_{n}(x)=-\lambda P_{n}(x)+P_{n+1}(0) s(x)+\lambda \sum_{k=1}^{n-1} P_{n-k}(x) g_{k}, \\
n=2, \cdots, N-1
\end{gathered}
$$

$-\frac{d}{d x} P_{n}(x)=-\lambda P_{n}(x)+P_{n+1}(0) s(x)$

$$
+\lambda \sum_{k=1}^{n-1} P_{n-k}(x) g_{k}+U_{n}(0) s(x), n \geq N
$$

$-\frac{d}{d x} Q_{0}(x)=-\lambda Q_{0}(x)+P_{1}(0) v(x)$

$$
\begin{gathered}
-\frac{d}{d x} Q_{n}(x)=-\lambda Q_{n}(x)+\lambda \sum_{k=1}^{n} Q_{n-k}(x) g_{k}, \\
n=1,2, \cdots
\end{gathered}
$$

$$
\begin{aligned}
-\frac{d}{d x} U_{N}(x)= & -\lambda U_{N}(x)+Q_{N}(0) u(x) \\
& +\lambda u(x) \sum_{k=0}^{N-1} R_{k}(x) g_{N-k}
\end{aligned}
$$

$$
\begin{aligned}
-\frac{d}{d x} U_{n}(x) & =-\lambda U_{n}(x)+\lambda \sum_{k=1}^{n-N} U_{n-k}(x) g_{k}+Q_{n}(0) u(x) \\
& +\lambda u(x) \sum_{k=0}^{N-1} R_{k} g_{n-k}, n=N+1, \cdots
\end{aligned}
$$

$$
\begin{aligned}
& 0=-\lambda R_{0}+Q_{0}(0) \\
& 0=-\lambda R_{n}+Q_{n}(0)+\lambda \sum_{k=1}^{n} R_{n-k} g_{k}, n=1, \cdots, N-1
\end{aligned}
$$

Taking the LST of both sides of Equations (4)-(10), we get

$$
\theta P_{1}^{*}(\theta)-P_{1}(0)=\lambda P_{1}^{*}(\theta)-P_{2}(0) S^{*}(\theta)
$$




$$
\begin{aligned}
& \theta P_{n}^{*}(\theta)-P_{n}(0)=\lambda P_{n}^{*}(\theta)-P_{n+1}(0) S^{*}(\theta) \\
&- \lambda \sum_{k=1}^{n-1} P_{n-k}^{*}(\theta) g_{k}, \\
& n=2, \cdots, N-1 \\
& \theta P_{n}^{*}(\theta)-P_{n}(0)=\lambda P_{n}^{*}(\theta)-P_{n+1}(0) S^{*}(\theta) \\
&-\lambda \sum_{k=1}^{n-1} P_{n-k}^{*}(\theta) g_{k}-U_{n}(0) S^{*}(\theta), \\
& n \geq N \\
& \theta Q_{0}^{*}(\theta)-Q_{0}(0)=\lambda Q_{0}^{*}(\theta)-P_{1}(0) V^{*}(\theta) \\
& \theta Q_{n}^{*}(\theta)-Q_{n}(0)=\lambda Q_{n}^{*}(\theta)-\lambda \sum_{k=1}^{n} Q_{n-k}^{*}(\theta) g_{k}, \\
& n=1,2, \cdots \\
& \theta U_{N}^{*}(\theta)-U_{N}(0)=\lambda U_{N}^{*}(\theta)-Q_{N}(0) \hat{U}^{*}(\theta) \\
&-\lambda \hat{U}^{*}(\theta) \sum_{k=0}^{N-1} R_{k} g_{N-k} \\
& \theta U_{n}^{*}(\theta)-U_{n}(0)=\lambda U_{n}^{*}(\theta)-\lambda \sum_{k=1}^{n-N} U_{n-k}^{*}(\theta) g_{k} \\
&-Q_{n}(0) \hat{U}^{*}(\theta)-\lambda \hat{U}^{*}(\theta) \sum_{k=0}^{N-1} R_{k} g_{n-k}, \\
& n=N+1, \cdots
\end{aligned}
$$

Noted that the LST of $P_{n}(x), Q_{n}(x)$ and $U_{n}(x)$ is defined as follows:

$$
\begin{gathered}
P_{n}^{*}(\theta)=\int_{0}^{\infty} e^{-\theta x} P_{n}(x) d x, \quad Q_{n}^{*}(\theta)=\int_{0}^{\infty} e^{-\theta x} Q_{n}(x) d x, \\
U_{n}^{*}(\theta)=\int_{0}^{\infty} e^{-\theta x} U_{n}(x) d x .
\end{gathered}
$$

Now, we define the following p.d.f.

$$
\begin{gathered}
P^{*}(z, \theta)=\sum_{n=1}^{\infty} P_{n}^{*}(\theta) z^{n}, \quad P(z, 0)=\sum_{n=1}^{\infty} P_{n}(0) z^{n} \\
Q^{*}(z, \theta)=\sum_{n=0}^{\infty} Q_{n}^{*}(\theta) z^{n}, Q(z, 0)=\sum_{n=0}^{\infty} Q_{n}(0) z^{n} \\
U^{*}(z, \theta)=\sum_{n=N}^{\infty} U_{n}^{*}(\theta) z^{n}, Q(z, 0)=\sum_{n=0}^{\infty} Q_{n}(0) z^{n} \\
R(z)=\sum_{n=0}^{N-1} R_{n} z^{n} .
\end{gathered}
$$

From Equations (13)-(15), we have

$$
\begin{aligned}
& {[\theta-\lambda+\lambda X(z)] P^{*}(z, \theta)=P(z, 0)} \\
& -S^{*}(\theta)\left[\frac{P(z, 0)}{z}-P_{1}(0)\right]-S^{*}(\theta) U(z, 0)
\end{aligned}
$$

Letting $\theta=\lambda-\lambda X(z)$, we get

$$
P(z, 0)=\frac{z S^{*}[\lambda-\lambda X(z)]\left[U(z, 0)-P_{1}(0)\right]}{z-S^{*}[\lambda-\lambda X(z)]}
$$

Substituting (21) into Equation (20), we have

$$
P^{*}(z, \theta)=\frac{z\left\{S^{*}[\lambda-\lambda X(z)]-S^{*}(\theta)\right\}\left[U(z, 0)-P_{1}(0)\right]}{\left\{z-S^{*}[\lambda-\lambda X(z)]\right\}[\theta-\lambda+\lambda X(z)]}
$$

Similarly, from (16), (17) and (18), (19) respectively and combining (11) and (12), we have

$$
\begin{aligned}
& Q(z, 0)=P_{1}(0) V^{*}[\lambda-\lambda X(z)] \\
& Q^{*}(z, \theta)=\frac{P_{1}(0)\left\{V^{*}[\lambda-\lambda X(z)]-V^{*}(\theta)\right\}}{\theta-\lambda+\lambda X(z)} \\
& U(z, 0)= \hat{U}^{*}[\lambda-\lambda X(z)] \\
& \cdot[Q(z, 0)+\lambda[X(z)-1] R(z)] \\
& U^{*}(z, \theta)=\left\{\hat{U}^{*}[\lambda-\lambda X(z)]-U^{*}(\theta)\right\} \\
& \times \frac{[Q(z, 0)+\lambda[X(z)-1] R(z)]}{\theta-\lambda+\lambda X(z)}
\end{aligned}
$$

Let $P(z)$ be the p.g.f. of the queue size at an arbitrary time epoch. Then,

$$
P(z)=P^{*}(z, 0)+Q^{*}(z, 0)+U^{*}(z, 0)+R(z)
$$

Using Equations (21)-(26) in $P(z)$, we have

$$
\begin{aligned}
P(z) & =\frac{(z-1) S^{*}[\lambda-\lambda X(z)]}{z-S^{*}[\lambda-\lambda X(z)]} \\
& \times\left\{\frac{1-V^{*}[\lambda-\lambda X(z)] \hat{U}^{*}[\lambda-\lambda X(z)]}{\lambda-\lambda X(z)} P_{1}(0)\right. \\
& \left.+\hat{U}^{*}[\lambda-\lambda X(z)] R(z)\right\}
\end{aligned}
$$

Following Lee et al. [7], we have

$$
R(z)=\frac{1}{\lambda} P_{1}(0) \sum_{n=0}^{N-1} \Psi_{n} z^{n}
$$

where $\Psi_{0}=\alpha_{0}=V^{*}(\lambda), \Psi_{n}=\sum_{i=0}^{n} \alpha_{i} \pi_{n-i}, n \geq 1, \pi_{0}=1$, and $\pi_{n}=\sum_{i=1}^{n} g_{i} \pi_{n-i}, n \geq 1 . \pi_{n}$ is the probability that system state visit $n$ during an idle period in the $\mathrm{M}^{\mathrm{x}} / \mathrm{G} / 1$ / N-policy queue [5]. 
Taking (29) into (28), we have

$$
\begin{aligned}
P(z) & =\frac{(z-1) S^{*}[\lambda-\lambda X(z)]}{z-S^{*}[\lambda-\lambda X(z)]} P_{1}(0) \\
& \times\left\{\frac{1-V^{*}[\lambda-\lambda X(z)] \hat{U}^{*}[\lambda-\lambda X(z)]}{\lambda-\lambda X(z)}\right. \\
& \left.+\frac{\hat{U}^{*}[\lambda-\lambda X(z)] \sum_{n=0}^{N-1} \Psi_{n} z^{n}}{\lambda}\right\}
\end{aligned}
$$

From Equation (30) and $P(1)=1$, we get

$$
P_{1}(0)=\frac{\lambda(1-\rho)}{\lambda(E[U]+E[V])+\sum_{n=0}^{N-1} \Psi_{n}}
$$

Thus, the p.d.f. of the system size distribution in the steady state becomes

$$
P(z)=G(z) \cdot \phi(z)
$$

where

$$
\begin{aligned}
& \phi(z)=\frac{1}{\lambda(E[U]+E[V])+\sum_{n=0}^{N-1} \Psi_{n}} \\
& \times\left\{\frac{1-V^{*}[\lambda-\lambda X(z)] \hat{U}^{*}[\lambda-\lambda X(z)]}{1-X(z)}\right. \\
& \left.+\hat{U}^{*}[\lambda-\lambda X(z)] \sum_{n=0}^{N-1} \Psi_{n} z^{n}\right\}
\end{aligned}
$$

\section{Remark 4.1}

Note that for $P(U=0)=1, E[U]=0$ and $\hat{U}^{*}[\lambda-$ $\lambda X(z)]=1$, then the Equation (32) agrees with Equation (25) of Lee et al. [7].

\section{Remark 4.2}

Let $N=1$, our model can be reduced to the batch arrival queue under a single vacation policy with startup. In this case the result (32) coincides with Equation (30) of Ke [21], in which the closedown time is assumed to be zero.

\section{The Queue Length Distribution}

\subsection{The Additional Queue Length Distribution}

This section, we will derive the additional queue length distribution. From (32), we see that the stationary queue length distribution of the $\mathrm{M}^{\mathrm{x}} / \mathrm{G} / 1$ queue under $\mathrm{N}$ policy with a single vacation and setup times decomposes into two independent random variables: one is the stationary queue length distribution of the ordinary $\mathrm{M}^{\mathrm{x}} / \mathrm{G} / 1$ queue; another is the additional queue length $\left(L^{a}\right)$ distribution due to $\mathrm{N}$ policy with a single vacation and setup times.

From the definition of p.d.f., the additional queue length distribution $p_{j}^{a}$ is given by

$$
p_{j}^{a}=P\left(L^{a}=j\right)=\left.\frac{1}{j !} \frac{d^{j}}{d z^{j}} \phi(z)\right|_{z=0}
$$

Obviously, the probability of additional queue length being zero is as follows

$$
p_{0}^{a}=\left.\phi(z)\right|_{z=0}=\frac{1}{\lambda(E[U]+E[V])+\sum_{n=0}^{N-1} \Psi_{n}}
$$

When $j=1, \cdots, N-1$, we can get the probability distribution of the additional queue length $L^{a}=j$ by direct derivative of $\phi(z)$.

$$
\begin{aligned}
p_{j}^{a} & =\frac{1}{\lambda(E[U]+E[V])+\sum_{n=0}^{N-1} \Psi_{n}} \frac{1}{j !} \frac{d^{j}}{d z^{j}} \\
& \times\left\{\frac{1-V^{*}[\lambda-\lambda X(z)] \hat{U}^{*}[\lambda-\lambda X(z)]}{1-X(z)}\right. \\
& \left.+\hat{U}^{*}[\lambda-\lambda X(z)] \sum_{n=0}^{N-1} \Psi_{n} z^{n}\right\}\left.\right|_{z=0}
\end{aligned}
$$

Following Leibniz formula, we have

$$
\begin{aligned}
p_{j}^{a} & =\frac{1}{\lambda(E[U]+E[V])+\sum_{n=0}^{N-1} \Psi_{n}} \frac{1}{j !} \sum_{k=0}^{j}\left(\begin{array}{l}
j \\
k
\end{array}\right)\left\{\frac{d^{k}}{d z^{k}}\left(\frac{1}{1-X(z)}\right)\right. \\
& \times \frac{d^{j-k}}{d z^{j-k}}\left(1-V^{*}[\lambda-\lambda X(z)] \hat{U}^{*}[\lambda-\lambda X(z)]\right) \\
& \left.+\frac{d^{k}}{d z^{k}} \hat{U}^{*}[\lambda-\lambda X(z)] \frac{d^{j-k}}{d z^{j-k}}\left(\sum_{n=0}^{N-1} \Psi_{n} z^{n}\right)\right\}\left.\right|_{z=0}, \\
& j=1, \cdots, N-1
\end{aligned}
$$

First, let us define the following functions $f_{x}(z)=$ $\frac{1}{1-X(z)}, f_{V}(z)=V^{*}[\lambda-\lambda X(z)]$ and $f_{\hat{U}}(z)=\hat{U}^{*}$. $[\lambda-\lambda X(z)]$. For sake of convenience, we note $\left.\frac{d^{k}}{d z^{k}} f_{X}(z)\right|_{z=0}=f_{x}^{(k)}(0)$. It is to be noted that the following recursion expressions hold. 


$$
\begin{aligned}
& \frac{f_{x}^{(n)}(0)}{n !}=\sum_{k=0}^{n-1} \frac{f_{x}^{(k)}(0)}{k !} \cdot g_{n-k}, \quad n \geq 1 \\
& \frac{f_{\hat{U}}^{(n)}(0)}{n !}=\int_{0}^{\infty} e^{-\lambda t} \frac{y^{(n)}(0, t)}{n !} d U(t), \quad n \geq 1 \\
& \frac{f_{V}^{(n)}(0)}{n !}=\int_{0}^{\infty} e^{-\lambda t} \frac{y^{(n)}(0, t)}{n !} d V(t), \quad n \geq 1
\end{aligned}
$$

where

$$
\begin{gathered}
\frac{y^{(n)}(0, t)}{n !}=\lambda t \sum_{k=0}^{n-1} \frac{k+1}{n} \cdot g_{k+1} \cdot \frac{y^{(n-k-1)}(0, t)}{(n-k-1) !}, \\
y(z, t)=e^{\lambda t X(z)} .
\end{gathered}
$$

Substitute (37)-(39) into (36), after some manipulations, we have

$$
\begin{gathered}
p_{j}^{a}=\frac{\sum_{k=0}^{j-1}-\frac{f_{X}^{(k)}(0)}{k !} \sum_{s=0}^{j-k} \frac{f_{V}^{(s)}(0)}{s !} \frac{f_{\hat{U}}^{(j-k-s)}(0)}{(j-k-s) !}}{\lambda(E[U]+E[V])+\sum_{n=0}^{N-1} \Psi_{n}} \\
+\frac{\frac{f_{X}^{(j)}(0)}{j !}\left(1-V *(\lambda) \hat{U}^{*}(\lambda)\right)+\sum_{k=0}^{j} \frac{f_{\hat{U}}^{(k)}(0)}{k !} \Psi_{j-k}}{\lambda(E[U]+E[V])+\sum_{n=0}^{N-1} \Psi_{n}} \\
j=1, \cdots, N-1
\end{gathered}
$$

When $j=N, N+1, \cdots$, in the similar manner proceeding with (36), we get the following additional queue length distribution.

$$
\begin{aligned}
& p_{j}^{a}=\frac{\sum_{k=0}^{j-1}-\frac{f_{X}^{(k)}(0)}{k !} \sum_{s=0}^{j-k} \frac{f_{V}^{(s)}(0)}{s !} \frac{f_{\hat{U}}^{(j-k-s)}(0)}{(j-k-s) !}}{\lambda(E[U]+E[V])+\sum_{n=0}^{N-1} \Psi_{n}} \\
& +\frac{\frac{f_{X}^{(j)}(0)}{j !}\left(1-V *(\lambda) \hat{U}^{*}(\lambda)\right)+\sum_{k=0}^{N-1} \Psi_{k} \frac{f_{\hat{U}}^{(j-k)}(0)}{(j-k) !}}{\lambda(E[U]+E[V])+\sum_{n=0}^{N-1} \Psi_{n}} \\
& j=N, N+1, \cdots
\end{aligned}
$$

With a direct method, we have derived the additional queue length distribution presented by (34), (40) and (41). Also, following the stochastic decomposition (32), the analytical queue length distribution can be derived.

\subsection{The Queue Length Distribution in Equilibrium}

Let $p_{j}=P\{L=j\}$ be the steady state probability distribution of queue length $(L)$ for the $\mathrm{M}^{\mathrm{x}} / \mathrm{G} / 1$ queueing system under N-policy with a single vacation and setup times. From (32), (1) and (34), we have

$$
p_{0}=\left.P(z)\right|_{z=0}=(1-\rho) \frac{1}{\lambda(E[U]+E[V])+\sum_{n=0}^{N-1} \Psi_{n}}
$$

When $j=1, \cdots, N-1$, the probability of queue length $L=j$ is given by

$$
\begin{aligned}
p_{j} & =P(L=j)=\left.\frac{1}{j !} \sum_{k=0}^{j}\left(\begin{array}{l}
j \\
k
\end{array}\right) \frac{d^{k} G(z)}{d z^{k}} \cdot \frac{d^{j-k} \phi(z)}{d z^{j-k}}\right|_{z=0} \\
& =\sum_{k=0}^{j} p_{k}^{0} \cdot p_{j-k}^{a}
\end{aligned}
$$

where $p_{j-k}^{a}$ is given by (40).

When $j=N, N+1, \cdots$, we first analyze the following two cases:

1) If $k \leq j-N$, that is $j-k \geq N, p_{j-k}^{a}$ is given by (41).

2) If $k \geq j-N+1$, that is $j-k \leq N-1, \quad p_{j-k}^{a}$ is given by (40).

So, the probability of queue length $L=j(j \geq N)$ is given by

$$
p_{j}=\sum_{k=0}^{j} p_{k}^{0} \cdot p_{j-k}^{a}=\sum_{k=0}^{j-N} p_{k}^{0} \cdot p_{j-k}^{a}+\sum_{k=j-N+1}^{j} p_{k}^{0} \cdot p_{j-k}^{a}
$$

The recursion expressions (42), (43) and (44) present the steady state queue length distribution for the $\mathrm{M}^{\mathrm{x}} / \mathrm{G} / 1$ queueing system under $\mathrm{N}$-policy with a single vacation and setup times. We can see that linking $\theta_{j}$ with (37)(39) the queue length distribution could be calculated by these recursion expressions.

\section{Numerical Experiments}

This section we try to illustrate the application of these recursion expressions by taking several numerical examples. Here we assume that the batch size distribution is displaced geometric distribution i.e. $g_{k}=(1-p) p^{k-1}$, $k \geq 1$. From (37), we have $f_{x}^{(n)}(0) / n !=1-p, n \geq 1$, which can be derived by the mathematical induction. The service time distributions are assumed to follow the exponential distribution with mean $E[S]=1 / \mu$. Furthermore, we take the vacation time distributions and setup time distributions are 3-stage Erlang distribution with finite mean $E[V]=3 / v$ and 4-stage Erlang distribution $E[U]=4 / u$ respectively. For the sake of convenience, we take $p=0.5, \lambda=2$ and $\mu=10$. We will investigate the effects of $N, v$ and $u$ on the queue length distribution with constant other system parameters. 
We present the results of the queue length distribution in Tables 1-3 with different $N, u$ and $v$. Observing the Table 1, it is clear that: 1) the probability of the system being empty $\left(\mathrm{P}_{0}\right)$ decreases as $N$ increases; 2) when $N$ is constant, the probability increases from $\mathrm{P}_{1}$ to $\mathrm{P}_{3}$ and then decreases and converges to zero.

Table 1. Queue length distribution vs the threshold $N$.

\begin{tabular}{|c|c|c|c|c|c|c|}
\hline \multicolumn{7}{|c|}{$u=10, v=12$} \\
\hline$N$ & 4 & 6 & 8 & 10 & 12 & 14 \\
\hline $\mathrm{P}_{0}$ & 0.1944 & 0.1504 & 0.1227 & 0.1036 & 0.0897 & 0.0791 \\
\hline $\mathrm{P}_{1}$ & 0.1469 & 0.1137 & 0.0928 & 0.0783 & 0.0678 & 0.0598 \\
\hline $\mathrm{P}_{2}$ & 0.1515 & 0.1172 & 0.0956 & 0.0808 & 0.0699 & 0.0616 \\
\hline $\mathrm{P}_{3}$ & 0.1526 & 0.1181 & 0.0964 & 0.0814 & 0.0704 & 0.0621 \\
\hline $\mathrm{P}_{4}$ & 0.0960 & 0.1180 & 0.0963 & 0.0813 & 0.0704 & 0.0620 \\
\hline $\mathrm{P}_{5}$ & 0.0720 & 0.1175 & 0.0958 & 0.0809 & 0.0701 & 0.0618 \\
\hline $\mathrm{P}_{6}$ & 0.0531 & 0.0732 & 0.0953 & 0.0805 & 0.0696 & 0.0614 \\
\hline $\mathrm{P}_{7}$ & 0.0387 & 0.0544 & 0.0946 & 0.0799 & 0.0692 & 0.0610 \\
\hline $\mathrm{P}_{8}$ & 0.0279 & 0.0398 & 0.0586 & 0.0795 & 0.0688 & 0.0606 \\
\hline $\mathrm{P}_{9}$ & 0.0200 & 0.0288 & 0.0433 & 0.0791 & 0.0684 & 0.0603 \\
\hline $\mathrm{P}_{10}$ & 0.0142 & 0.0206 & 0.0316 & 0.0488 & 0.0681 & 0.0601 \\
\hline $\mathrm{P}_{11}$ & 0.0102 & 0.0148 & 0.0229 & 0.0361 & 0.0680 & 0.0600 \\
\hline $\mathrm{P}_{12}$ & 0.0073 & 0.0106 & 0.0165 & 0.0264 & 0.0419 & 0.0598 \\
\hline $\mathrm{P}_{13}$ & 0.0053 & 0.0075 & 0.0118 & 0.0191 & 0.0310 & 0.0598 \\
\hline $\mathrm{P}_{14}$ & 0.0039 & 0.0054 & 0.0084 & 0.0137 & 0.0227 & 0.0368 \\
\hline $\mathrm{P}_{15}$ & 0.0031 & 0.0039 & 0.0060 & 0.0098 & 0.0164 & 0.0273 \\
\hline $\mathrm{P}_{16}$ & 0.0026 & 0.0029 & 0.0043 & 0.0070 & 0.0118 & 0.0199 \\
\hline $\mathrm{P}_{17}$ & 0.0022 & 0.0023 & 0.0032 & 0.0050 & 0.0085 & 0.0144 \\
\hline $\mathrm{P}_{18}$ & 0.0020 & 0.0019 & 0.0023 & 0.0036 & 0.0061 & 0.0104 \\
\hline $\mathrm{P}_{19}$ & 0.0018 & 0.0017 & 0.0019 & 0.0026 & 0.0044 & 0.0075 \\
\hline $\mathrm{P}_{20}$ & 0.0017 & 0.0015 & 0.0016 & 0.0020 & 0.0031 & 0.0053 \\
\hline EL & 3.3376 & 4.0702 & 4.8892 & 5.7465 & 6.6072 & 7.7492 \\
\hline sum & 0.2660 & 0.2693 & 0.4023 & 0.4126 & 0.4192 & 0.4216 \\
\hline
\end{tabular}


Table 2. Queue length distribution vs the parameter $u$.

\begin{tabular}{|c|c|c|c|c|c|c|}
\hline \multicolumn{7}{|c|}{$N=6, v=12$} \\
\hline$u$ & 10 & 11 & 12 & 13 & 14 & 15 \\
\hline $\mathrm{P}_{0}$ & 0.1504 & 0.1523 & 0.1539 & 0.1552 & 0.1564 & 0.1575 \\
\hline $\mathrm{P}_{1}$ & 0.1137 & 0.1157 & 0.1175 & 0.1191 & 0.1204 & 0.1217 \\
\hline $\mathrm{P}_{2}$ & 0.1172 & 0.1190 & 0.1205 & 0.1219 & 0.1230 & 0.1240 \\
\hline $\mathrm{P}_{3}$ & 0.1181 & 0.1197 & 0.1210 & 0.1221 & 0.1231 & 0.1240 \\
\hline $\mathrm{P}_{4}$ & 0.1180 & 0.1194 & 0.1207 & 0.1217 & 0.1226 & 0.1234 \\
\hline$P_{5}$ & 0.1175 & 0.1188 & 0.1200 & 0.1210 & 0.1218 & 0.1226 \\
\hline $\mathrm{P}_{6}$ & 0.0732 & 0.0719 & 0.0707 & 0.0696 & 0.0686 & 0.0676 \\
\hline $\mathrm{P}_{7}$ & 0.0544 & 0.0529 & 0.0515 & 0.0503 & 0.0492 & 0.0483 \\
\hline $\mathrm{P}_{8}$ & 0.0398 & 0.0383 & 0.0371 & 0.0359 & 0.035 & 0.0341 \\
\hline $\mathrm{P}_{9}$ & 0.0288 & 0.0275 & 0.0264 & 0.0254 & 0.0246 & 0.0239 \\
\hline $\mathrm{P}_{10}$ & 0.0206 & 0.0195 & 0.0186 & 0.0179 & 0.0172 & 0.0166 \\
\hline $\mathrm{P}_{11}$ & 0.0148 & 0.0140 & 0.0133 & 0.0127 & 0.0122 & 0.0117 \\
\hline $\mathrm{P}_{12}$ & 0.0106 & 0.0099 & 0.0094 & 0.0089 & 0.0086 & 0.0082 \\
\hline $\mathrm{P}_{13}$ & 0.0075 & 0.0070 & 0.0067 & 0.0063 & 0.0061 & 0.0058 \\
\hline $\mathrm{P}_{14}$ & 0.0054 & 0.0051 & 0.0048 & 0.0045 & 0.0043 & 0.0042 \\
\hline $\mathrm{P}_{15}$ & 0.0039 & 0.0037 & 0.0035 & 0.0033 & 0.0032 & 0.0030 \\
\hline $\mathrm{P}_{16}$ & 0.0029 & 0.0027 & 0.0026 & 0.0024 & 0.0023 & 0.0023 \\
\hline $\mathrm{P}_{17}$ & 0.0023 & 0.0022 & 0.002 & 0.0019 & 0.0019 & 0.0018 \\
\hline $\mathrm{P}_{18}$ & 0.0019 & 0.0018 & 0.0017 & 0.0016 & 0.0016 & 0.0015 \\
\hline $\mathrm{P}_{19}$ & 0.0017 & 0.0016 & 0.0015 & 0.0014 & 0.0013 & 0.0013 \\
\hline $\mathrm{P}_{20}$ & 0.0015 & 0.0014 & 0.0013 & 0.0013 & 0.0012 & 0.0012 \\
\hline EL & 4.0702 & 3.9953 & 3.9327 & 3.8797 & 3.8343 & 3.7948 \\
\hline sum & 0.2693 & 0.3783 & 0.3711 & 0.3644 & 0.3591 & 0.3541 \\
\hline
\end{tabular}


Table 3. Queue length distribution vs the parameter $v$.

\begin{tabular}{|c|c|c|c|c|c|c|}
\hline \multicolumn{7}{|c|}{$N=6, u=10$} \\
\hline$v$ & 10 & 11 & 12 & 13 & 14 & 15 \\
\hline $\mathrm{P}_{0}$ & 0.1482 & 0.1494 & 0.1504 & 0.1513 & 0.152 & 0.1527 \\
\hline $\mathrm{P}_{1}$ & 0.1126 & 0.1132 & 0.1137 & 0.1140 & 0.1142 & 0.1144 \\
\hline $\mathrm{P}_{2}$ & 0.1172 & 0.1173 & 0.1172 & 0.1171 & 0.117 & 0.1168 \\
\hline $\mathrm{P}_{3}$ & 0.1184 & 0.1183 & 0.1181 & 0.1179 & 0.1178 & 0.1176 \\
\hline $\mathrm{P}_{4}$ & 0.1182 & 0.1181 & 0.1180 & 0.1179 & 0.1178 & 0.1178 \\
\hline $\mathrm{P}_{5}$ & 0.1175 & 0.1175 & 0.1175 & 0.1175 & 0.1175 & 0.1175 \\
\hline $\mathrm{P}_{6}$ & 0.0736 & 0.0734 & 0.0732 & 0.0731 & 0.0730 & 0.0729 \\
\hline $\mathrm{P}_{7}$ & 0.0547 & 0.0545 & 0.0544 & 0.0542 & 0.0541 & 0.0540 \\
\hline $\mathrm{P}_{8}$ & 0.0401 & 0.0399 & 0.0398 & 0.0396 & 0.0396 & 0.0395 \\
\hline $\mathrm{P}_{9}$ & 0.0291 & 0.0289 & 0.0288 & 0.0287 & 0.0286 & 0.0285 \\
\hline $\mathrm{P}_{10}$ & 0.0209 & 0.0207 & 0.0206 & 0.0205 & 0.0204 & 0.0204 \\
\hline $\mathrm{P}_{11}$ & 0.0151 & 0.0149 & 0.0148 & 0.0147 & 0.0146 & 0.0146 \\
\hline $\mathrm{P}_{12}$ & 0.0109 & 0.0107 & 0.0106 & 0.0105 & 0.0104 & 0.0103 \\
\hline $\mathrm{P}_{13}$ & 0.0079 & 0.0077 & 0.0075 & 0.0074 & 0.0074 & 0.0073 \\
\hline $\mathrm{P}_{14}$ & 0.0057 & 0.0056 & 0.0054 & 0.0053 & 0.0053 & 0.0052 \\
\hline $\mathrm{P}_{15}$ & 0.0042 & 0.0041 & 0.0039 & 0.0038 & 0.0038 & 0.0037 \\
\hline $\mathrm{P}_{16}$ & 0.0032 & 0.0030 & 0.0029 & 0.0028 & 0.0027 & 0.0027 \\
\hline $\mathrm{P}_{17}$ & 0.0026 & 0.0025 & 0.0023 & 0.0022 & 0.0021 & 0.0021 \\
\hline $\mathrm{P}_{18}$ & 0.0022 & 0.0021 & 0.0019 & 0.0018 & 0.0018 & 0.0017 \\
\hline $\mathrm{P}_{19}$ & 0.0020 & 0.0018 & 0.0017 & 0.0016 & 0.0015 & 0.0014 \\
\hline $\mathrm{P}_{20}$ & 0.0018 & 0.0016 & 0.0015 & 0.0014 & 0.0013 & 0.0013 \\
\hline EL & 4.1328 & 4.0969 & 4.0702 & 4.0500 & 4.0343 & 4.0220 \\
\hline sum & 0.2740 & 0.2714 & 0.2693 & 0.2676 & 0.2666 & 0.2656 \\
\hline
\end{tabular}


From Tables 2-3, one sees that 1) the probability of the system being empty increases as $u$ or $v$ increases; 2) the probability $\mathrm{P}_{j}(1 \leq j \leq N-1)$ increases as $u$ or $v$ increases, while the probability $\mathrm{P}_{j}(j \geq N)$ decreases as $u$ or $v$ increases; 3 ) the probability $\mathrm{P}_{j}$ increases from $\mathrm{P}_{1}$ to $\mathrm{P}_{3}$ and then decreases and converges to zero under constant $u$ or $v$.

The mean queue length (EL) is also presented in Tables 1-3 respectively. It is shown that as $N$ increases mean queue length increases, while mean queue length decreases as $u$ or $v$ increases. It is worth mentioning that the mean queue length could not being the only one performance measure for the system design and optimization. For example, when $N=8, u=10, v=12$, the mean queue length is 4.8892 , but the total of the probability (sum) when the queue length exceeding 5 is 0.4023 , which could not be neglected.

\section{Conclusions}

In this paper we have derived the additional queue length distribution and the recursion expressions of the queue length distribution for the $\mathrm{M}^{\mathrm{x}} / \mathrm{G} / 1$ queueing system under $\mathrm{N}$-policy with a single vacation and setup times. Furthermore, we present the numerical results of the queue length distribution and the distribution properties are also investigated. The results in this paper would be significant and useful to system designers and others. The approach developed in this paper is powerful and can be used to analyze more complex queueing system.

\section{References}

[1] B. T. Doshi, "Queueing Systems with Vacations-a Survey,” Queueing System, Vol. 1, No. 1, 1986, pp. 29-66.

[2] H. Takagi, "Queueing Analysis: A Foundation of Performance Evaluation, Vacation and Priority System,” Elsevier, Amsterdam, Vol. 1, 1991.

[3] S. W. Fuhrmann and R. B. Cooper, "Stochastic Decompositions in the M/G/1 Queue with Generalized Vacations,” Operations Research, Vol. 33, No. 5, 1985, pp. 1117-1129.

[4] M. Yadin and P. Naor, "Queueing Systems with a Removable Service Station,” Operational Research Quarterly, Vol. 14, No. 3, 1963, pp. 393-405.

[5] H. W. Lee, S. S. Lee and K. C. Chae, "Operating Characteristics of $\mathrm{M}^{\mathrm{x}} / \mathrm{G} / 1$ Queue with N-Policy,” Queueing Systems, Vol. 15, No. 1-4, 1994, pp. 387-399.

[6] H. W. Lee, S. S. Lee, J. O. Park and K. C. Chae, “Analysis of $\mathrm{M}^{\mathrm{x}} / \mathrm{G} / 1$ Queue with N Policy and Multiple Vacations,” Journal of Applied Probability, Vol. 31, No. 2, 1994, pp. 467-496.

[7] S. S. Lee, H. W. Lee, S. H. Yoon and K. C. Chae,
"Batch Arrival Queue with N Policy and Single Vacation,” Computers and Operations Research, Vol. 22, No. 2, 1995, pp. 173-189.

[8] G. Choudhury and M. Paul, "A Batch Arrival Queue with an Additional Service Channel under N-Policy,” Applied Mathematics and Computation, Vol. 156, No. 1, 2004, pp. 115-130.

[9] G. Choudhury and K. C. Madan, “A Two-Stage Batch Arrival Queueing System with a Modified Bernoulli Schedule Vacation under N-Policy," Mathematical and Computer Modelling, Vol. 42, No. 1-2, 2005, pp. 71-85.

[10] G. Choudhury and M. Paul, "A Batch Arrival Queue with a Second Optional Service Channel under N-Policy,” Stochastic Analysis and Applications, Vol. 24, No. 1, 2006, pp. 1-21.

[11] J.-C. Ke, "The Control Policy of an $M^{\mathrm{x}} / \mathrm{G} / 1$ Queueing System with Server Startup and Two Vacation Types," Mathematical Methods of Operations Research, Vol. 54, No. 3, 2001, pp. 471-490.

[12] J.-C. Ke, "Optimal Strategy Policy in Batch Arrival Queue with Server Breakdowns and Multiple Vacations," Mathematical Methods of Operations Research, Vol. 58, No. 1, 2003, pp. 41-56.

[13] R. G. V. Krishna, R. Nadarajan and R. Arumuganathan, "Analysis of a Bulk Queue with N-Policy Multiple Vacations and Setup Times," Computers and Operations Research, Vol. 25, No. 11, 1998, pp. 957-967.

[14] K.-H. Wang, M.-C. Chan and J.-C. Ke, "Maximum Entropy Analysis of the $\mathrm{M}^{\mathrm{x}} / \mathrm{M} / 1$ Queueing System with Multiple Vacations and Server Breakdowns," Computers \& Industrial Engineering, Vol. 52, No. 2, 2007, pp. 192202.

[15] J. E. Shore, "Information Theoretic Approximations for M/G/1 and G/G/1 Queueing Systems,” Acta Informatica, Vol. 17, No.1, 1982, pp. 43-61.

[16] M. A. El-Affendi and D. D. Kouvatsos, “A Maximum Entropy Analysis of the M/G/1 and G/M/1 Queueing Systems at Equilibrium,” Acta Informatica, Vol. 19, No. 4, 1983, pp. 339-355.

[17] J.-C. Ke and C.-H. Lin, "Maximum Entropy Solutions for Batch Arrival Queue with an Unreliable Server and Delaying Vacations,” Applied Mathematics and Computation, Vol. 183, No. 2, 2006, pp. 1328-1340.

[18] Y. H. Tang, "The Transient Solution for M/G/1 Queue with Server Vacations," Acta Mathematica Scientia, Vol. 17, No. 3, 1997, pp. 276-282.

[19] Y. H. Tang and X. W. Tang, "The Queue-Length Distribution for $\mathrm{M}^{\mathrm{X}} / \mathrm{G} / 1$ Queue with Single Server Vacation,” Acta Mathematica Scientia, Vol. 20, No. 3, 2000, pp. 397-408.

[20] Y. H. Tang, X. Yun and S. J. Huang, "Discrete-Time Geo $/ G / 1$ Queue with Unreliable Server and Multiple Adaptive Delayed Vacations,” Journal of Computational and Applied Mathematics, Vol. 220, No. 1-2, 2008, pp. 439-455.

[21] J.-C. Ke, "Batch Arrival Queues under Vacation Policies with Server Breakdowns and Startup/Closedown Times," Applied Mathematical Modelling, Vol. 31, No. 7, 2007, pp. 1282-1292. 